\title{
CLAVES EMPÍRICAS DE LA SATISFACCIÓN Y DEL COMPROMISO DEL TALENTO EN LAS ORGANIZACIONES
}

\author{
Empirical talent satisfaction and engagement \\ keys in organizations
}

Justo Villafañe

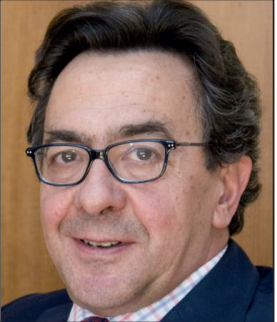

Resumen

Dentro del marco teórico de la teoría de la reputación corporativa, se identifican empíricamente las claves de las que depende la satisfacción de los empleados en una organización y su compromiso con ésta. Los estudios sobre gestión del talento consideran muchas veces que un alto porcentaje de los empleados de una compañía están satisfechos con su trabajo porque el índice de satisfacción de su clima interno puede llegar incluso al $80 \%$. Sin embargo, uno de los primeros objetivos que este estudio se planteó fue verificar empíricamente no sólo el número de empleados satisfechos sino el de los comprometidos, ya que lograr la evolución desde organizaciones satisfechas hacia organizaciones comprometidas es el reto que plantea la teoría de la reputación corporativa en su aplicación al mundo de la dirección de personas y de la gestión del talento en las organizaciones.

\section{Palabras clave}

Reputación corporativa; Gestión del talento; Calidad laboral; Marca empleador; Reputación interna; Satisfacción; Compromiso.

\section{Abstract}

In this study we identify the key components of employee satisfaction and level of commitment to their jobs using the theoretical framework of corporate reputation theory. Recent studies in talent management have analyzed satisfaction and consider $80 \%$ to be a high number. However, this study looks not only at satisfaction, but also employee commitment. According to theory of reputation, companies need to have employees that are both satisfied and committed.

\section{Keywords}

Corporate reputation; Talent management; Work quality; Employer branding; Internal reputation; Satisfaction; Engagement.

Villafañe, Justo (2017). "Claves empíricas de la satisfacción y del compromiso del talento en las organizaciones". El profesional de la información, v. 26, n. 6, pp. 1159-1170.

https://doi.org/10.3145/epi.2017.nov.15

\section{Introducción}

El presente estudio pretende esclarecer, de manera empírica, las claves de las que depende el compromiso de los empleados con su empresa. Tradicionalmente no se ha distinguido entre personas satisfechas y personas comprometidas en la gestión del talento en las organizaciones. Esta gestión se basa en la mayor parte de las compañías en los estudios de clima interno, los cuales analizan la satisfacción de una plantilla en función de un número indeterminado de variables. Estos estudios indican, no sólo el grado de satisfacción del conjunto de los trabajadores, sino el tipo de intervención que se debe abordar para corregir las variables cuyos valores son críticos y, supuestamente, mejorar el grado de compromiso con la organización.

Frente a la práctica habitual de intervenir variable a variable para tratar de elevar los valores críticos, lo que esta investi- 
gación viene a demostrar es que para aumentar el grado de compromiso de una plantilla debe partirse de la satisfacción como un todo -como una gestalt- y actuar holísticamente para que la satisfacción mejore en su conjunto, no en unas pocas variables, y que los empleados satisfechos asciendan al escalón del compromiso. Este es el denominado reto del compromiso: alcanzar el mayor número de empleados comprometidos ya que en la actualidad sólo el $41 \%$ lo están, según los datos que figuran en este estudio basado en una muestra que recoge las valoraciones de 17.555 trabajadores que participaron en el ranking de Merco Talento 2015 de las 100 mejores empresas para trabajar en España.

\section{El estudio pretende esclarecer las claves de las que depende el compromiso de los empleados con su empresa}

El corpus teórico desde el que se aborda esta investigación es el de la teoría de la reputación corporativa. Esta disciplina está presente en los planes de estudio de numerosas universidades en España y en el mundo, y ha tenido en las últimas dos décadas un importante desarrollo debido al valor creciente de los recursos intangibles de las empresas. Desde la óptica de la reputación se pueden revisar, o reinterpretar, la mayor parte de estos recursos intangibles de las organizaciones como son la marca, el liderazgo, la gobernanza..., o el talento.

Aunque este estudio se haya planteado desde la perspectiva reputacional del talento y, como ya se ha dicho, está fundamentado principalmente en la teoría de la reputación corporativa, era absolutamente necesario no sólo efectuar una revisión honda sobre la doctrina académica de la gestión del talento sino, y muy especialmente, de las aportaciones de aquellos autores (Gratton, 2003; Villafañe, 2006; Alonso; García-Muina, 2014) que han aproximado en sus trabajos ambos paradigmas teóricos, el de la reputación y el de la gestión del talento. Tras esta revisión teórica se plantearon las siguientes preguntas de investigación, a las que el presente estudio ha tratado de responder y de las que se infieren las correspondientes hipótesis y objetivos del estudio. En la verificación empírica se emplearon diversas técnicas de segmentación con el fin de analizar los diferentes tipos de trabajadores y sus comportamientos para encontrar modelos predictivos y pautas de intervención. A continuación de cada pregunta e hipótesis de investigación se indica la técnica estadística empleada:

1) ¿Son pertinentes las 15 variables de talento empleadas en la investigación y, en segundo lugar, existe correlación entre éstas y la satisfacción global? La hipótesis es afirmativa como respuesta a ambas preguntas. Para comprobarlo fue necesario efectuar un análisis de correlación que verificara ambas hipótesis y, de ser así, estableciese el grado de correlación de cada una de las variables de talento.

2) ¿Se puede atribuir una mayor influencia en la satisfacción de los empleados al valor reputación interna que a los otros dos -marca empleador y calidad laboral- y especialmente con relación a este último? La hipótesis sustentada es que las variables reputacionales -de naturaleza más intangibleson las más determinantes de la satisfacción y del compromiso. La técnica utilizada fue la frecuencia media.

3) ¿Se puede categorizar una tipología de trabajadores en función de su comportamiento en la organización inferido a partir del grado de satisfacción con cada uno de las 15 variables de talento? La hipótesis no sólo es favorable, sino que mediante un análisis cluster es posible establecer el perfil de cada uno de esos tipos de empleados incluyendo, entre otros hallazgos, las variables de talento que definen el comportamiento del talento comprometido.

4) ¿Existen otros factores diferentes de la satisfacción que puedan explicar el comportamiento del talento en una organización? La hipótesis es de difícil pronóstico sin efectuar un análisis factorial o de componentes principales.

5) ¿Se puede determinar el peso de cada variable de talento en la satisfacción global? La hipótesis es afirmativa y el análisis de regresión múltiple, resuelve fácilmente esta pregunta permitiendo medir el peso de cada variable.

6) ¿Qué categorías permitirían definir los comportamientos laborales en función del peso de las variables de talento? ¿Podría dibujarse un mapa de percepción del talento que combinase las variables de éste y la tipología de empleados? También creemos que es posible asociar determinadas variables a ciertos comportamientos, comprobación que exige el empleo del análisis factorial de correspondencias.

7) ¿Se pueden establecer las variables de talento que determinan la adscripción de los empleados a cada tipo? El mismo análisis factorial de correspondencias confirmará esta última hipótesis.

\section{Marco teórico}

Como ya se ha dicho, el abordaje que aquí se propone proviene principalmente del corpus de la teoría de la reputación corporativa. Esta teoría se desarrolla en los noventa (Fombrun; Shanley, 1990; Fombrun; Van-Riel, 1997; 2003) y se consolida en el nuevo siglo, e incluso son superados muchos de esos planteamientos previos (Villafañe, 2012; Fombrun, 2012; Carreras; Alloza; Carreras, 2013). Walker (2010) realiza, a partir de Wartick (2002), una completa revisión bibliográfica puntualizando lo que, según este autor, dibuja el perímetro del concepto de reputación corporativa y las otras nociones que deben ser excluidas del mismo. Lange, Lee y Dai (2011) también valoran diferentes enfoques del concepto de reputación empresarial resumiéndolos en tres:

- la notoriedad de la empresa que implica suficiente información corporativa para un stakeholder que le permita adoptar decisiones con relación a sus intereses;

- las creencias y expectativas que se tienen de la empresa; - el atractivo global de la organización.

Existen también diversos enfoques epistemológicos de la reputación próximos a la teoría de la empresa. El primero es el de la teoría de recursos y capacidades (Roberts; Dowling, 2002; Martin-de-Castro; Navas-López; LópezSáez, 2006; McWilliams; Siegel, 2001; McWilliams; Siegel; Wright, 2006; Castelo-Branco; Lima-Rodrigues, 2006), para 
los cuales la reputación corporativa es uno de los recursos más valiosos de una empresa porque, igual que otros intangibles corporativos, no son imitables y, además, optimizan su relación la mayoría de sus grupos de interés. Un segundo enfoque economicista de la reputación es el de aquellos autores que la consideran una suerte de garantía contractual, determinante del comportamiento empresarial (Cornell; Shapiro, 1987; De-la-Fuente-Sabaté; De-Quevedo-Puente, 2003) y muy basada en la confianza, lo que rebajaría de manera notable los costes de transacción. Un tercer enfoque es el de identificación de la reputación corporativa con el capital social (Arregle et al., 2007) de una empresa que se favorecería de una amplísima red de relaciones y contactos positivos que redundan en sus resultados.

Junto a la teoría de la reputación corporativa, el segundo marco teórico de esta investigación lo constituye la gestión del talento. Éste es uno de los recursos intangibles de las organizaciones que mayor valor y competitividad puede aportar a las mismas en línea con las aportaciones académicas más representativas sobre el tema (Smart, 1999; Walker; Larocco, 2002; Lewis; Heckman, 2006; Villafañe, 2006; CoIlings; Mellahi 2009; Scullion; Collings; Caligiuri, 2010; Tarique; Schuler, 2010; Kehinde, 2012; Stahl et al., (2012).

Este enfoque reputacional del talento no es ajeno incluso a la evolución de la propia denominación utilizada para referirse al concurso humano en las organizaciones:

- del término "trabajo", o trabajadores u obreros, que era propio de la teoría económica clásica que lo consideraba, junto a la tierra y el capital, como uno de los factores básicos de la producción;

- a la utilización de "capital humano", o recursos humanos, como un estadio intermedio, ya dentro de las relaciones laborales organizadas;

- hasta la actualidad en que el término "talento", en línea con múltiples autores (Ulrich, 1996; Cubeiro, 2003; Lawler III, 2008; Ulrich et al., 2009; Gasalla, 2015), ha dejado de ser una aportación de valor impersonal a la organización para convertirse en uno de los factores que mayor valor añaden a una empresa y constituirse, por tanto, en una de las claves de su competitividad.

Desde el marco teórico que aquí se propone, la reputación de una empresa es el reconocimiento que hacen de su realidad corporativa sus principales grupos de interés en función del grado de satisfacción de las expectativas de éstos con relación al comportamiento de esa empresa (Villafañe, 2012). Uno de los grupos clave de cualquier compañía son sus empleados y, en tal sentido, el estudio de su relación con la organización no solamente no puede ser ajeno a la teoría de la reputación corporativa, sino que constituye una parte central de ésta. Veamos en primer lugar cuál es la perspectiva de la teoría de la reputación respecto al talento de las organizaciones para, ulteriormente, ahondar en la evolución de lo que se ha denominado el reto del compromiso (Gratton, 2003), es decir, la evolución desde organizaciones satisfechas hacia organizaciones comprometidas.

El clima interno es consecuencia de la calidad laboral, conditio sine qua non para la atracción y retención del talento; pero siendo la calidad laboral una condición necesaria, ya no es suficiente en los sectores e industrias de alto rendimiento, en los que ya no discrimina positivamente porque lo que determina la elección de una organización para trabajar está cada vez más relacionado con factores más intangibles como la reputación de la compañía, la de su líder, la sostenibilidad o una marca que represente valores con los que las personas se identifican profesional y personalmente.

La primera característica, y la que mejor define a una empresa desde la perspectiva de la teoría de la reputación corporativa, es su naturaleza multi stakeholder (Freeman, 1984). La reputación proporciona legitimación a la empresa (Bartlett; Pallas; Frostenson, 2013, pp. 530-544) a partir de una actitud proactiva respecto hacia sus stakeholders que se concreta en su habilidad para mantener una relación empática con ellos, satisfacer sus demandas y crear valor compartido. Dentro de esta diversidad de stakeholders, los empleados son uno de los más importantes (Cheese; Thomas; Craig, 2008; Caplan, 2011; Davies; Kourdi, 2010), probablemente sean los más decisivos, tras los clientes, o en ocasiones por delante de éstos (Nayar, 2010).

\section{Lo que determina la elección de una or- ganización para trabajar está cada vez más relacionado con factores más intan- gibles como la reputación de la compa- ñía, la de su líder, la RSC o una marca que represente valores con los que las per- sonas se identifican profesional y perso- nalmente}

Consecuentemente, si para la empresa sus empleados son clave, la valoración que éstos hagan de su organización será igualmente determinante y aquellas que cuenten con una mejor reputación interna gozarán también de un mayor grado de compromiso y de vinculación del talento que llevan a bordo. En este sentido, la reputación interna se define de la misma manera que la corporativa pero teniendo en cuenta a un solo stakeholder: los empleados. La reputación interna se definiría, por tanto, como el reconocimiento que hacen los empleados del comportamiento de su empresa con relación al conjunto de sus grupos de interés (Villafañe, 2006, p. 25), y no sólo a ellos mismos, porque si fuera así estaríamos hablando de calidad laboral, concepto que conviene deslindar del de reputación interna.

Existe una tercera noción, que cada vez se relaciona más con la calidad laboral y con la reputación interna, aunque también es diferente, es la denominada marca empleador o, dicho de otra manera, la imagen que una organización tiene exteriormente como un lugar apetecible para trabajar (Barrow; Mosley, 2005; Brown; Turner, 2008; Jiménez; Aguado 2009; Burchell; Robin, 2011); un hecho que, no por ubicarse extra muros de la organización, deja de tener menos importancia que los dos anteriormente citados como lo demuestran los esfuerzos que las grandes compañías hacen para lograr atraer y comprometer al mejor talento en sus organizaciones. 
Por tanto, nos encontramos que en la gestión del talento desde la perspectiva reputacional aparecen tres valores: la calidad laboral, la reputación interna y la marca empleador, que delimitan el mapa de la satisfacción de las personas en el seno de una organización (gráfico 1).

Si la gestión clásica del talento la constituían las personas y la definición de sus puestos de trabajo, de cuya adecuación dependía en gran medida la satisfacción y la calidad laboral de una plantilla, en el presente $-y$ desde la perspectiva reputacional de la dirección de personas- es necesario añadir dos nuevas dimensiones:

- la reputación interna, que se deriva de la importancia que hoy se les reconoce a los miembros de una organización como uno de los stakeholders más determinantes de su reputación corporativa y,

- el reconocimiento que la propia compañía, a través de su marca corporativa, es capaz de inducir para que se considere una marca empleador atractiva y deseada para trabajar.

Este texto pretende constituirse como una primera aproximación al talento desde la teoría de la reputación corporativa y, quizá sea éste uno de sus méritos aspiracionales: superar la brecha respecto a la bibliografía científica anterior que provenía de las teorías de la reputación o de la dirección de personas, porque los integra ambos. La cuestión que cabe plantear a continuación es si resulta suficiente una buena reputación interna, una calidad laboral satisfactoria y una marca atractiva hacia el exterior para garantizar la satisfacción y el compromiso de los trabajadores de una organización. La respuesta no es unívoca, sino que suscita muchas otras cuestiones, algunas de las cuales trataremos de responder en las siguientes páginas basándonos, no sólo en opiniones propias o de otros autores sino, y casi exclusivamente, en evidencias empíricas resultado de un trabajo estadístico exhaustivo.

\section{Metodología}

La base empírica de esta investigación se encuentra en los resultados del estudio Merco Talento 2015, publicado en octubre de 2016 y que recoge los resultados del ejercicio ce-

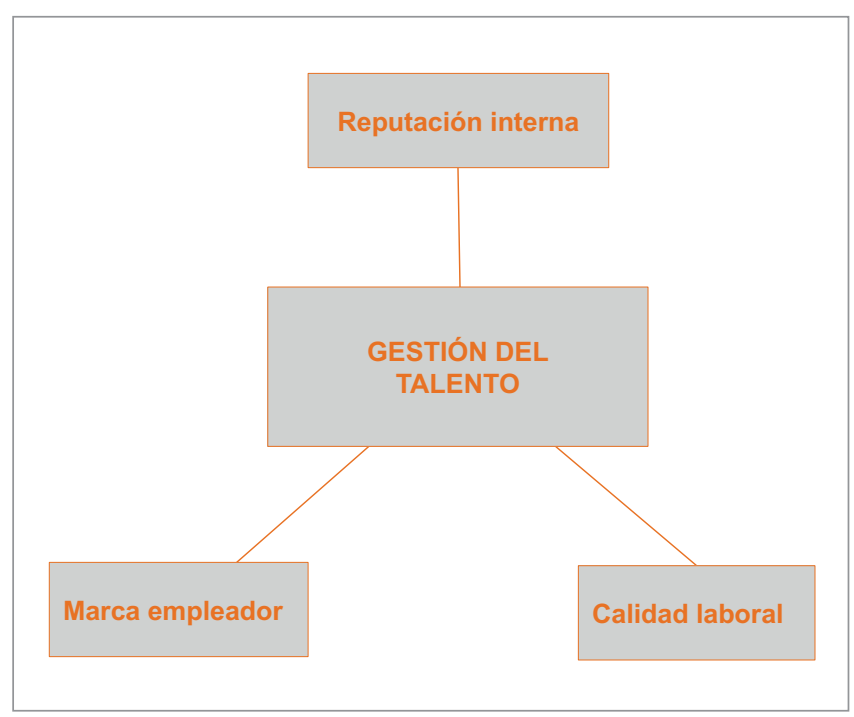

Gráfico 1. Componentes de la gestión del talento desde la teoría de la reputación corporativa

rrado anual. El estudio incluye, entre otras, las valoraciones de una muestra de 18.458 trabajadores en España que evalúan a sus propias empresas, y a las cien de Merco Empresas en función de los tres valores de talento antes citados y de las 15 variables en las que éstos se desagregan y que explican las claves de atracción y vinculación del talento en las organizaciones (tabla 1).

Merco Talento (http://www.merco.info) es un monitor que evalúa anualmente las mejores empresas para trabajar en diversos países, entre ellos España, en donde se viene realizando desde 2006, cuando se publicó por vez primera el ranking con Las 100 mejores empresas para trabajar en España, que utiliza una metodología, a nuestro juicio, muy rigurosa y cuyos resultados, así como el proceso de toma de datos, son verificados por la auditora KPMG de acuerdo con la norma ISAE 3000. Según el estudio de la publicación especializada Capital humano (Carazo-Muriel, 2015, pp. 36-39) Merco Talento fue considerado el mejor monitor de empleo de los elaborados y difundidos en España (tabla 2).

Tabla 1. Valores y variables del talento

\begin{tabular}{|l|l|l|}
\hline Calidad laboral & Marca empleador & Reputación interna \\
\hline Salario & Sector atractivo & Valores éticos y profesionales \\
\hline Desarrollo profesional & Admirada por valores y resultados & lgualdad y diversidad \\
\hline Motivación y reconocimiento & Capacidad para atraer talento & Liderazgo de alta dirección \\
\hline Relación con los mandos intermedios & Recomendación & Identificación con el proyecto empresarial \\
\hline Conciliación & Acreditación y reconocimiento & Orgullo de pertenencia \\
\hline
\end{tabular}

Tabla 2. Comparativa de monitores de empleo elaborados y difundidos en España

\begin{tabular}{|l|c|c|c|}
\hline & Conocimiento & $\begin{array}{c}\text { Percepción de } \\
\text { utilidad actual }\end{array}$ & $\begin{array}{c}\text { Proyección de } \\
\text { utilidad futuro }\end{array}$ \\
\hline Great Place to Work & 6,84 & 3,37 & 3,69 \\
\hline Merco Talento & 6,48 & 3,30 & 3,70 \\
\hline Top Employers & 4,56 & 2,66 & 3,13 \\
\hline Rankings de Empleadores Ideales & 2,29 & 2,23 & 2,86 \\
\hline Investor in People IIP & 1,78 & 2,03 & 5,38 \\
\hline
\end{tabular}


Independientemente de las valoraciones externas, como las que hacen las publicaciones especializadas en la dirección de personas, la razón por la que se ha elegido Merco Talento, monitor que hasta 2014 se denominó Merco Personas, es su metodología, la cual le convierte en una fuente de información muy rigurosa y útil para esta investigación porque aporta una base muestral de una gran robustez (gráfico 2).

A partir de esta muestra, el objeto del presente estudio es identificar empíricamente los factores que determinan la satisfacción y el clima interno de una empresa y establecer, a partir de ahí, las claves para lograr el compromiso de los trabajadores con la organización como el desiderátum de la vinculación del talento a una compañía; no en vano hay autores relevantes en este ámbito que han definido el talento como la capacidad basada en el compromiso (Ulrich, 1996).

\section{Resultados}

El primero e importante resultado convalida la selección de variables empleada en el estudio y demuestra la elevada correlación entre la satisfacción y las citadas variables de talento. El análisis de correlación efectuado confirma que todas las correlaciones entre satisfacción y talento son altas y significativas, alcanzando un tercio de dichas variables una correlación superior a 0,80 (el máximo valor es 1 ), dentro de las

Tabla 3. Correlación entre la satisfacción y las variables de talento

\begin{tabular}{|l|c|}
\hline \multicolumn{1}{|c|}{ Resumen de correlaciones } & Máxima correlación \\
\hline Orgullo de pertenencia & $\mathbf{0 , 8 2 6}$ \\
\hline Motivación y reconocimiento & $\mathbf{0 , 8 1 1}$ \\
\hline Reconocimientos como buen empleador & $\mathbf{0 , 8 0 5}$ \\
\hline Identificación con el proyecto empresarial & $\mathbf{0 , 8 0 4}$ \\
\hline Recomendada por los que la conocen & $\mathbf{0 , 8 0 3}$ \\
\hline Valores éticos y profesionales & 0,785 \\
\hline Liderazgo alta dirección & 0,770 \\
\hline Desarrollo profesional & 0,753 \\
\hline Atracción de talento & 0,749 \\
\hline Admirada por valores, gestión y resultados & 0,739 \\
\hline Igualdad y diversidad & 0,702 \\
\hline Salario coherente con la función desempeñada & 0,675 \\
\hline Conciliación y beneficios sociales & 0,665 \\
\hline Relación con los mandos inmediatos & 0,654 \\
\hline Sector atractivo para trabajar & 0,627 \\
\hline Valoración global & $\mathbf{1 , 0 0 0}$ \\
\hline
\end{tabular}

cuales el orgullo de pertenencia $(0,82)$ y la motivación y el reconocimiento $(0,81)$ obtienen la mayor correlación (tabla 3).

\section{El análisis empírico efectuado permite establecer que el $59 \%$ de los empleados de las mejores empresas para trabajar en España no se encuentran comprome- tidos con ellas}

En España la satisfacción global media de los trabajadores con sus empresas es 7,66; apreciándose una diferencia significativamente menor entre las variables correspondientes al valor calidad laboral $(7,28)$ con relación a los otros dos valores, la reputación interna $(7,90)$ y la marca empleador $(7,79)$ confirmando la hipótesis 2 . En cuanto a las variables más críticas, son también tres de las relacionadas con la calidad laboral las que ofrecen brechas estadísticamente significativas:

- salario $(6,96)$,

- motivación y reconocimiento $(6,97), y$

- desarrollo profesional $(7,15)$.

El orgullo de pertenencia $(8,27)$ es, por el contrario, la variable mejor valorada en cuanto a la satisfacción global de los trabajadores en sus empresas (gráfico 3).

Estos resultados, fruto de un análisis de correlación, vienen a confirmar uno de los presupuestos de la investigación, ya expuesto en la introducción: que la calidad laboral es una condición necesaria para atraer y mantener al talento pero no determinante para logar una vinculación que garantice el compromiso de las personas con sus empresas, menor que aquellos factores, como el citado orgullo de pertenencia, más intangibles y de naturaleza reputacional. 


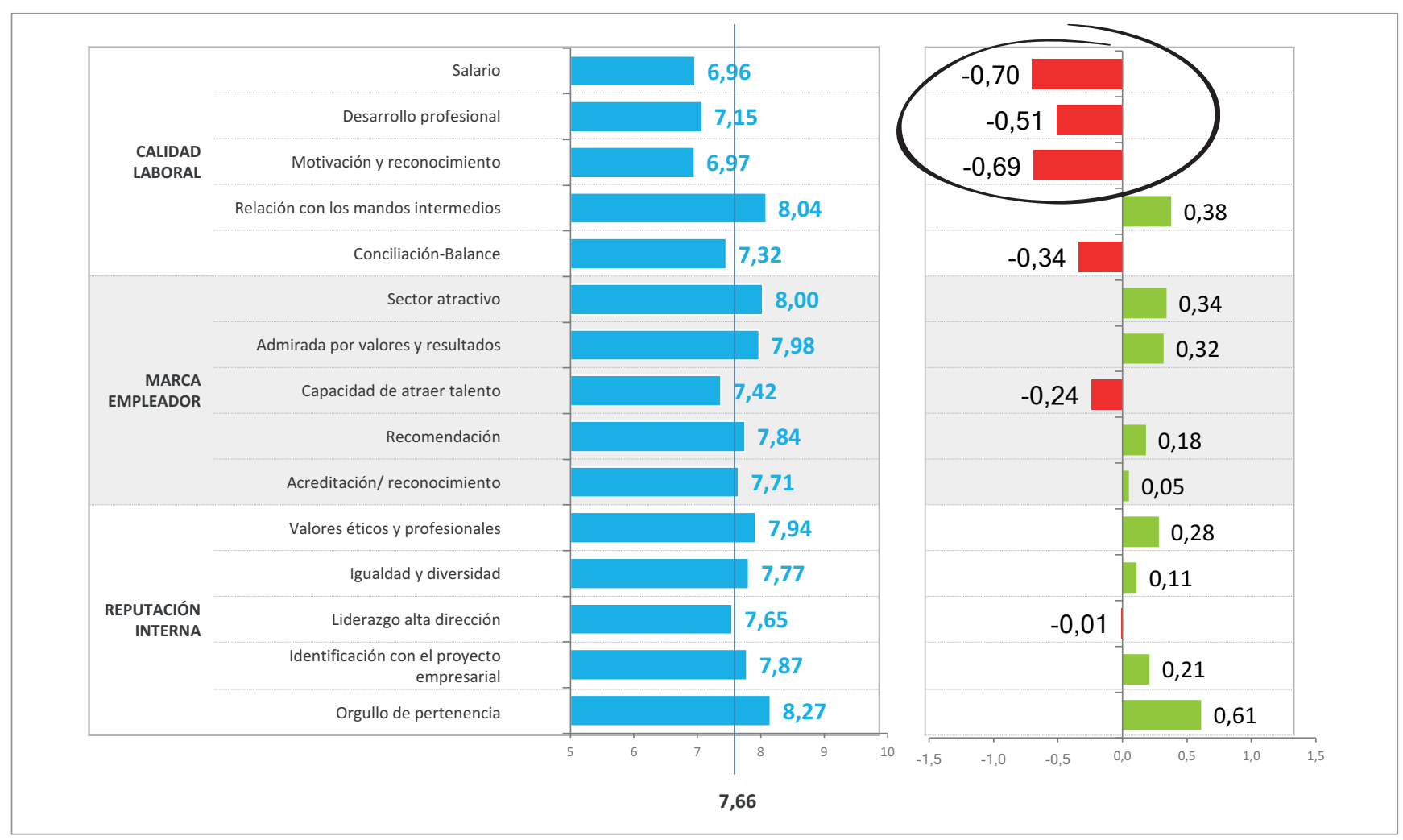

Gráfico 3. Satisfacción global media de los trabajadores en España y gaps respecto a la media

Otro resultado concluyente es que cuanta menor categoría profesional tiene un trabajador menor satisfacción experimenta; alcanzando diferencias notables en casi todas las variables entre la categoría más baja -la de empleado u operario- con relación a las otras tres, tal como se observa en las valoraciones de la siguiente tabla. No todos los trabajadores que respondieron los cuestionarios indicaron su categoría profesional, esa es la razón de que las medias no coincidan con el total (tabla 4). Efectivamente, en 13 de las 15 variables de talento la satisfacción de los empleados menos cualificados es la más baja entre las cuatro categorías profesionales, lo que establece una relación directa entre satisfacción y formación, ya que la valoración media de las variables aumenta correlativamente con la categoría profesional.

Tabla 4. Satisfacción global por categorías profesionales. En rojo se indican los valores de menor satisfacción

\begin{tabular}{|c|c|c|c|c|c|c|}
\hline & \multirow{2}{*}{\multicolumn{5}{|c|}{ Categoría profesional }} \\
\hline & & & & & & \\
\hline & & Total & $\begin{array}{l}\text { Director o } \\
\text { responsable del } \\
\text { área funcional }\end{array}$ & $\begin{array}{l}\text { Mando inter- } \\
\text { medio }\end{array}$ & $\begin{array}{l}\text { Profesional cua- } \\
\text { lificado / técnico } \\
\text { especialista }\end{array}$ & $\begin{array}{c}\text { Empleado / } \\
\text { operario }\end{array}$ \\
\hline \multirow{5}{*}{$\begin{array}{l}\text { Calidad } \\
\text { laboral }\end{array}$} & Salario & 6,96 & 7,39 & 6,92 & 6,99 & 7,15 \\
\hline & Desarrollo profesional & 7,15 & 8,02 & 7,31 & 6,90 & 6,82 \\
\hline & Motivación y reconocimiento & 6,97 & 7,78 & 7,13 & 6,83 & 6,71 \\
\hline & Relación con mandos intermedios & 8,04 & 8,50 & 8,15 & 8,03 & 7,81 \\
\hline & Conciliación & 7,32 & 7,42 & 7,24 & 7,64 & 7,58 \\
\hline \multirow{5}{*}{$\begin{array}{l}\text { Marca } \\
\text { empleador }\end{array}$} & Sector atractivo & 8,00 & 8,30 & 8,05 & 8,13 & 7,96 \\
\hline & Admirada por valores, gestión y resultados & 7,98 & 8,42 & 8,05 & 8,05 & 7,91 \\
\hline & Capacidad de atraer talento & 7,42 & 7,89 & 7,46 & 7,41 & 7,35 \\
\hline & Recomendación & 7,84 & 8,29 & 7,90 & 7,88 & 7,86 \\
\hline & Acreditación/reconocimiento & 7,71 & 8,18 & 7,76 & 7,77 & 7,67 \\
\hline \multirow{5}{*}{$\begin{array}{l}\text { Reputación } \\
\text { interna }\end{array}$} & Valores éticos y profesionales & 7,94 & 8,48 & 8,03 & 7,95 & 7,78 \\
\hline & Igualdad y diversidad & 7,77 & 8,22 & 7,87 & 7,79 & 7,64 \\
\hline & Liderazgo alta dirección & 7,65 & 8,26 & 7,70 & 7,59 & 7,61 \\
\hline & Identificación con el proyecto empresarial & 7,87 & 8,49 & 7,97 & 7,82 & 7,79 \\
\hline & Orgullo de pertenencia & 8,27 & 8,76 & 8,36 & 8,27 & 8,26 \\
\hline \multicolumn{2}{|r|}{ Valoración global } & 7,70 & 8,19 & 7,75 & 7,71 & 7,71 \\
\hline
\end{tabular}


Un nuevo resultado, muy útil para los objetivos del estudio, ha sido la formalización de una tipología de trabajadores en función de su comportamiento en la organización, confirmando plenamente la hipótesis 3. A partir de un análisis cluster se agrupan los trabajadores con comportamientos similares (en este caso la referencia es el individuo) y se segmentan las variables en función de las que más diferencian cada comportamiento (en este segundo caso la referencia es la muestra) (tabla 5).

Aunque en la tabla anterior lo que más llame la atención sean las cifras críticas: más de un $20 \%$ de los trabajadores están desvinculados emocionalmente de su organización, o en deserción, lo que resulta central para los fines de este estudio es el porcentaje del $38,5 \%$ de las personas satisfechas, pero no identificadas o comprometidas con su empresa. Este es el reto fundamental en la gestión del talento desde la teoría de la reputación corporativa, encontrar fórmulas que permitan evolucionar desde una plantilla satisfecha hacia una plantilla comprometida.

Independientemente de cómo se valoren estos resultados es necesario tener en cuenta que la muestra de análisis de esta investigación está formada por trabajadores de las empresas con mejor reputación corporativa en España, es decir, de la élite empresarial española, en la que presumiblemente algunos valores del talento como la calidad laboral o incluso la marca empleador son de los más elevados del país y en este contexto es en el que los citados datos tipológicos cobran todo su sentido. A nuestro juicio, que sólo el $41 \%$ de las personas estén comprometidas con su organización es un hecho que justifica por sí sólo el gran reto que tienen por delante las empresas, el reto del compromiso (tabla 6).

La hipótesis 4 del estudio no se pudo confirmar ya que mediante un intento de análisis factorial, o análisis de componentes principales, se trató de identificar algunos ejes básicos o dimensiones que agruparan variables o atributos referidos a ideas parecidas y que, al mismo tiempo, dichas dimensiones fueran independientes entre sí. Veamos un ejemplo de análisis de componentes principales en un estudio de clima interno en el que se tuvieron en cuenta tres factores o dimensiones: las oportunidades de carrera en la empresa, el apoyo de los jefes a la carrera de los emplea-

\begin{tabular}{|c|c|c|c|c|}
\hline Comprometidos & Satisfechos & Desvinculados & En deserción & Total \\
\hline 8,25 & 6,77 & 5,06 & 3,47 & 6,96 \\
\hline 8,64 & 6,97 & 4,92 & 2,77 & 7,15 \\
\hline 8,64 & 6,78 & 4,48 & 2,01 & 6,97 \\
\hline 9,17 & 7,91 & 6,42 & 4,45 & 8,04 \\
\hline 8,69 & 7,12 & 5,44 & 3,02 & 7,32 \\
\hline 9,08 & 7,81 & 6,59 & 4,84 & 8,01 \\
\hline 9,22 & 7,83 & 6,34 & 3,71 & 7,98 \\
\hline 8,87 & 7,22 & 5,41 & 2,89 & 7,42 \\
\hline 9,21 & 7,72 & 5,94 & 2,95 & 7,84 \\
\hline 9,12 & 7,57 & 5,74 & 2,80 & 7,71 \\
\hline 9,27 & 7,83 & 6,11 & 3,09 & 7,94 \\
\hline 9,11 & 7,63 & 5,89 & 3,41 & 7,77 \\
\hline 9,09 & 7,51 & 5,64 & 2,61 & 7,65 \\
\hline 9,24 & 7,78 & 5,96 & 2,83 & 7,87 \\
\hline 9,57 & 8,27 & 6,38 & 2,90 & 8,27 \\
\hline 9,01 & 7,51 & 5,75 & 3,18 & 7,66 \\
\hline 9,02 & 7,59 & 5,85 & 3,11 & 7,7 \\
\hline
\end{tabular}




\begin{tabular}{|c|c|c|c|c|c|}
\hline & Factor 1 & Factor 2 & Factor 3 & & \\
\hline $\begin{array}{l}\text { Creo que la empresa sabe retener a los buenos } \\
\text { profesionales }\end{array}$ & 0,80 & 0,13 & 0,16 & \multirow{4}{*}{ Factor 1} & \multirow{4}{*}{$\begin{array}{l}\text { Las } \\
\text { oportunidades } \\
\text { de carrera de la } \\
\text { empresa }\end{array}$} \\
\hline $\begin{array}{l}\text { Esta empresa ofrece oportunidades de promoción y } \\
\text { carrera }\end{array}$ & 0,79 & 0,28 & 0,24 & & \\
\hline $\begin{array}{l}\text { En la empresa se facilita que los empleados } \\
\text { desarrollen trabajos diferentes a lo largo de su } \\
\text { carrera }\end{array}$ & 0,71 & 0,29 & 0,24 & & \\
\hline $\begin{array}{l}\text { Creo que las posibilidades de desarrollo y promoción } \\
\text { son similares en todas las áreas / negocios de la } \\
\text { empresa }\end{array}$ & 0,69 & 0,21 & 0,29 & & \\
\hline Mi jefe apoya mi desarrollo profesional & 0,18 & 0,89 & 0,10 & \multirow[b]{2}{*}{ Factor 2} & \multirow{2}{*}{$\begin{array}{l}\text { El apoyo de los } \\
\text { jefes a la } \\
\text { carrera }\end{array}$} \\
\hline $\begin{array}{l}\text { mi responsable se preocupa y pone medios para que } \\
\text { las personas de su equipo se desarrollen }\end{array}$ & 0,35 & 0,79 & 0,14 & & \\
\hline $\begin{array}{l}\text { Las oportunidades de desarrollo que ofrece la } \\
\text { empresa... son las mismas para personas de cualquier } \\
\text { nacionalidad }\end{array}$ & 0,25 & 0,08 & 0,85 & \multirow[t]{2}{*}{ Factor 3} & \multirow{2}{*}{$\begin{array}{l}\text { La igualdad de } \\
\text { oportunidades } \\
\text { en la carrera }\end{array}$} \\
\hline $\begin{array}{l}\text { Las oportunidades de desarrollo que ofrece la } \\
\text { empresa... son las mismas entre hombres y mujeres }\end{array}$ & 0,26 & 0,15 & 0,83 & & \\
\hline
\end{tabular}

dos y la igualdad de oportunidades (tabla 7).

No fue posible el análisis factorial porque la satisfacción es el único factor o dimensión independiente con el cual correlacionan todas las variables analizadas en este informe y no aparecen otras dimensiones del trabajo con la suficiente independencia respecto a la satisfacción. Dicho de manera más simple, el estudio viene a confirmar empíricamente que la satisfacción lo explica todo.

A través de un análisis de regresión múltiple (ARM) se consiguió averiguar la importancia o el peso de las variables de talento en la satisfacción global, ya que el ARM es un modelo econométrico que permite saber cuánto pesan una serie de variables independientes en una variable dependiente, como es el caso de la satisfacción. Se confirmó, por tanto, la hipótesis 5 del estudio en cuyo resultado destacan el orgullo de pertenencia, un salario coherente con la función desempeñada y la motivación y el reconocimiento, como las tres variables de talento más importantes en la satisfacción global para los miembros de una organización (gráfico 4).

$Y$ las tres que menor importancia tienen: una empresa admirada por sus valores; la gestión y los resultados; la atracción del talento y la igualdad y diversidad (gráfico 5).

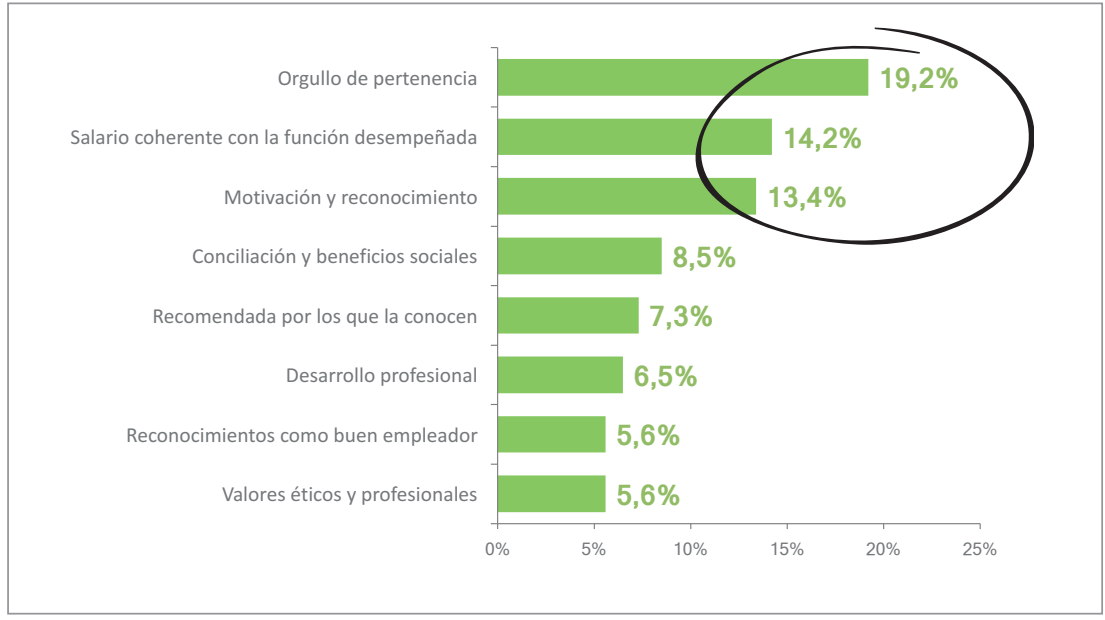

Gráfico 4. Las variables de talento con mayor importancia en la satisfacción

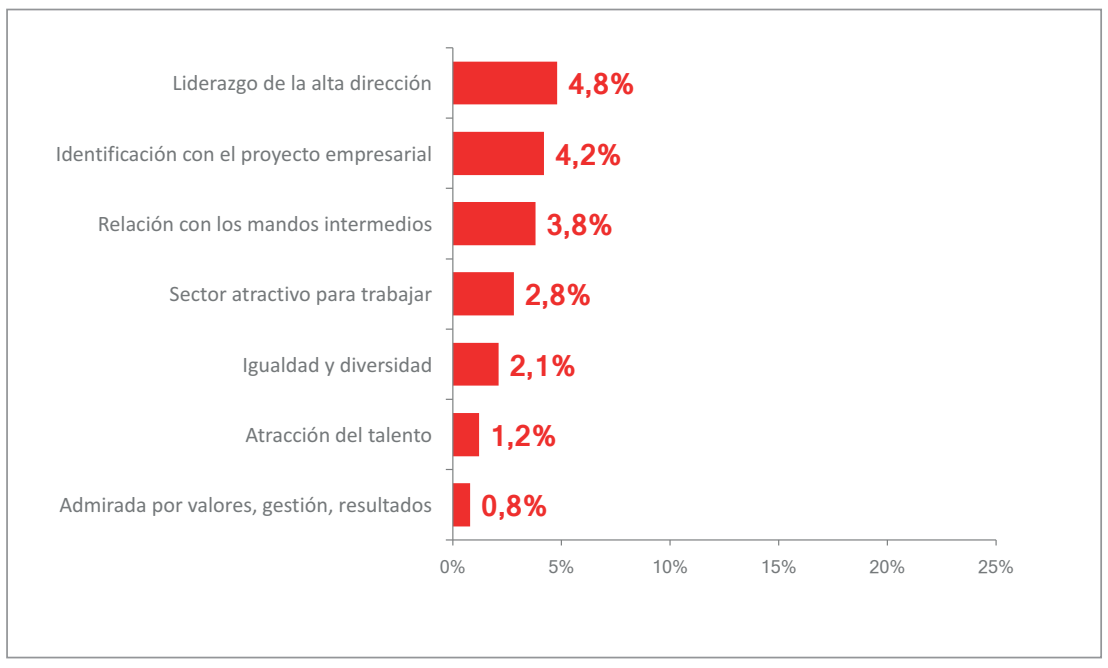

Gráfico 5. Las variables de talento con menor importancia en la satisfacción 


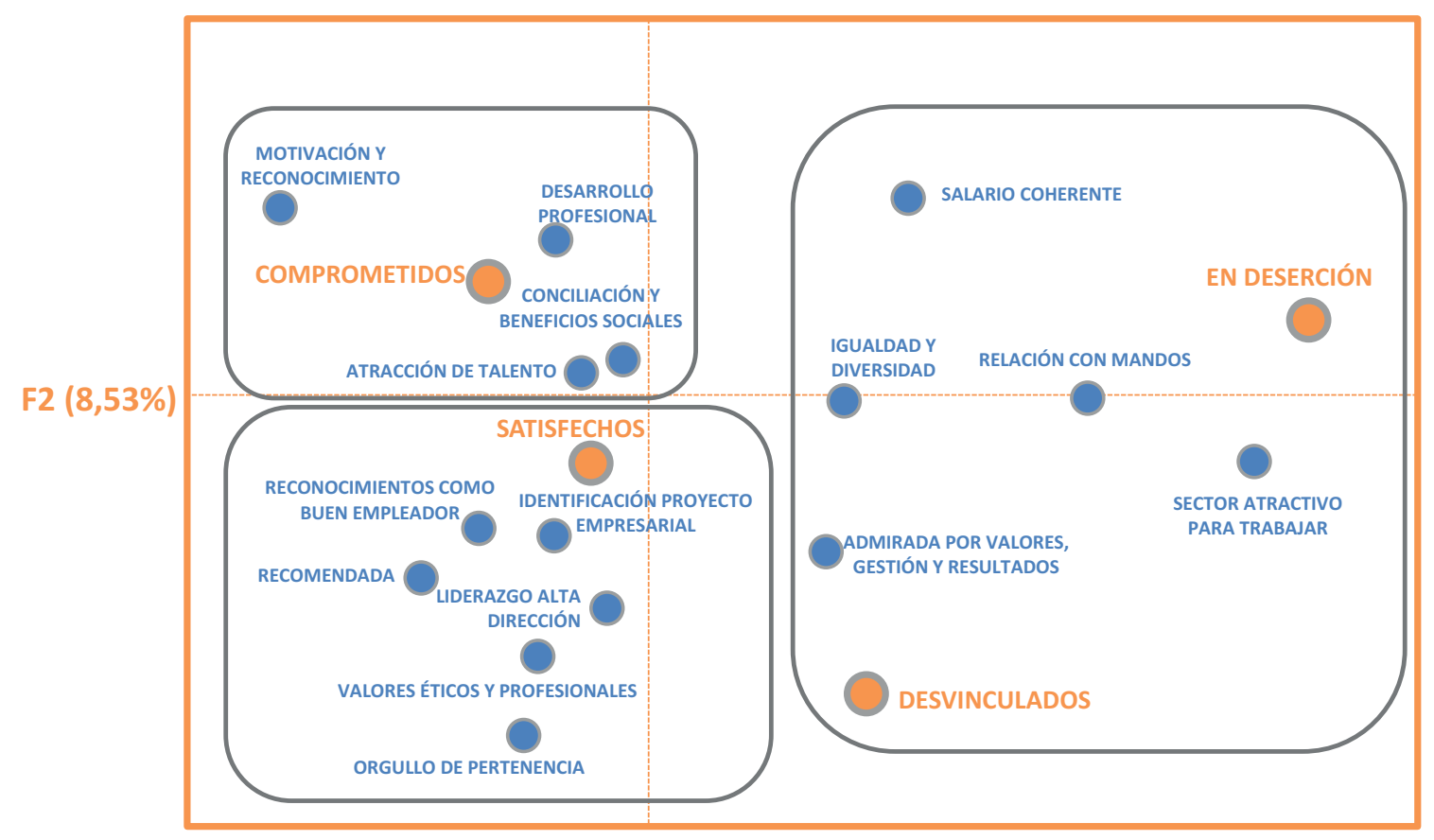

F1 $(91,29 \%)$

Gráfico 6. Mapa de percepción de variables de talento y tipos de empleados

Otro hallazgo del estudio ha sido la asociación entre las variables de talento y la tipología de trabajadores previamente formalizada. Para formalizar esta asociación se utilizó el análisis factorial de correspondencias (AFC) que es una técnica que considera los rasgos diferenciales de cada variable de talento con relación a las demás y permite establecer mapas de percepción. En dichos mapas de percepción se observan las variables correspondientes a cada tipo de trabajador. La varianza explicada de cada eje del mapa equivale al volumen de información que aporta. Queda también confirmada la hipótesis 6 de la investigación (gráfico 6).

\section{En las mejores empresas para trabajar en España un 40,9\% están comprome- tidos con su empresa; un $38,5 \%$ se en- cuentran satisfechos; un $16,3 \%$ desvin- culados y un $4,3 \%$ en deserción}

Además, el mismo AFC pudo establecer la correspondencia de variables por tipo, es decir, qué variables de talento determinan la adscripción de los trabajadores a un determinado tipo. En este sentido, existen unas variables de naturaleza funcional que son bien valoradas por todos los tipos de empleados. Otro grupo de las variables de talento se explican en términos de bienestar laboral, y sólo son bien valoradas por los empleados satisfechos y comprometidos y, finalmente, un tercer grupo de variables que producen identificación con el proyecto empresarial y que se corresponden, exclusivamente, con los trabajadores comprometidos o identificados con su empresa, el objeto de la hipótesis 7 que se confirma enteramente. Esta correspondencia entre las variables de talento y los tipos de trabajadores se representa gráficamente en la 'pirámide del compromiso' (gráfico 7).

\section{En España la satisfacción global media de los trabajadores con sus empresas es 7,66 en base 10 .}

\section{Discusión y conclusiones}

El análisis empírico efectuado hasta ahora permite establecer una primera conclusión que debería propiciar un replanteamiento de la gestión del talento en las organizaciones: que más de la mitad de los empleados de las mejores empresas para trabajar en España no se encuentran comprometidos con ellas: exactamente el 59\%.

En este nuevo planteamiento que implica la gestión reputacional del talento hay que partir casi axiomáticamente de que la satisfacción global que las personas experimentan en el desempeño de su trabajo en la organización no se puede descomponer, variable por variable, para tratar de optimizar dicha satisfacción mejorando aquellas variables de talento críticas en los estudios de clima interno porque la satisfacción es una gestalt que no tiene el mismo sentido si es analizada parte a parte, o variable a variable.

Los actuales estudios de clima sólo tienen validez o utilidad para elaborar lo que en el texto se ha denominado 'mapa de comportamiento', que asocia variables de talento a 
comportamientos organizacionales. A partir de estos mapas de comportamiento, los cuales delimitan los perfiles de los empleados desertores, desvinculados, satisfechos y comprometidos, habrá que plantear actuaciones globales que incumban al menos a los tres tipos de trabajadores (con los desertores lo más aconsejable es pactar su salida), actuaciones de naturaleza cualitativa que busquen, mediante el consenso, metas comunes y compromisos verificables (Villafañe, 2006) que, al margen de las condiciones contractuales, constituyan metas aspiracionales tanto para la dirección como para la plantilla de una empresa.

Según los resultados expuestos en el epígrafe anterior resulta empíricamente recomendable primar el orgullo de pertenencia y la motivación y reconocimiento ya que, según el análisis cluster realizado, el sentido de pertenencia es la variable con mayor puntuación entre los trabajadores comprometidos (9,6 en base 10). Ambas variables son también las que mayor correlación presentan con la satisfacción: el orgullo de pertenencia $(0,82)$ y la motivación y reconocimiento $(0,81)$ siendo 1 el máximo valor posible.

Las dos variables citadas -orgullo de pertenencia $(19,2 \%)$ y motivación y reconocimiento $(13,4 \%)$ - figuran también entre las tres más importantes según el análisis de regresión múltiple que se ha efectuado. Esta última, la motivación y el reconocimiento $(6,97)$, es la segunda más demandada por parte de los trabajadores en España, por encima incluso del salario $(6,96)$, siendo la media de las 15 variables de talento de 7,66 , lo que indica el fuerte componente aspiracional de este factor que, a diferencia de otras variables como el mismo salario o la conciliación, es mucho más accionable ya que no implica otros recursos que los meramente intangibles y actitudinales. Según el análisis factorial de correspondencias, la motivación y el reconocimiento son, además, el factor más diferenciador del tipo de los trabajadores comprometidos.

Los resultados de esta investigación vienen a confirmar los supuestos básicos provenientes de la teoría de la reputación -principalmente que son aquellas variables de naturaleza reputacional, como las ahora citadas del orgullo de pertenencia y la motivación y el reconocimiento- las que mayor poder de vinculación tienen con la organización y, también refutan algunos postulados de los enfoques clásicos de la gestión del talento...

El orgullo de pertenencia, la motivación y el reconocimiento constituyen las dos claves principales de los trabajadores satisfechos

El orgullo de pertenencia y la motivación y el reconocimiento constituyen, desde una perspectiva empírica, las dos claves principales no sólo de los trabajadores satisfechos, sino también los rasgos que mejor definen a las personas comprometidas con sus organizaciones y se convierten en objetivos operativos, muy concretos, para alcanzar el anhelado reto del compromiso.

El presente estudio apunta una nueva línea de investigación que podría denominarse la gestión reputacional del compromiso del talento en las organizaciones. Su principal hallazgo puede resumirse en que dos variables como el orgullo de pertenencia y la motivación y el reconocimiento se constituyen en las claves más determinantes para vincular y comprometer a las personas con su organización. Lo que la continuación de esta investigación

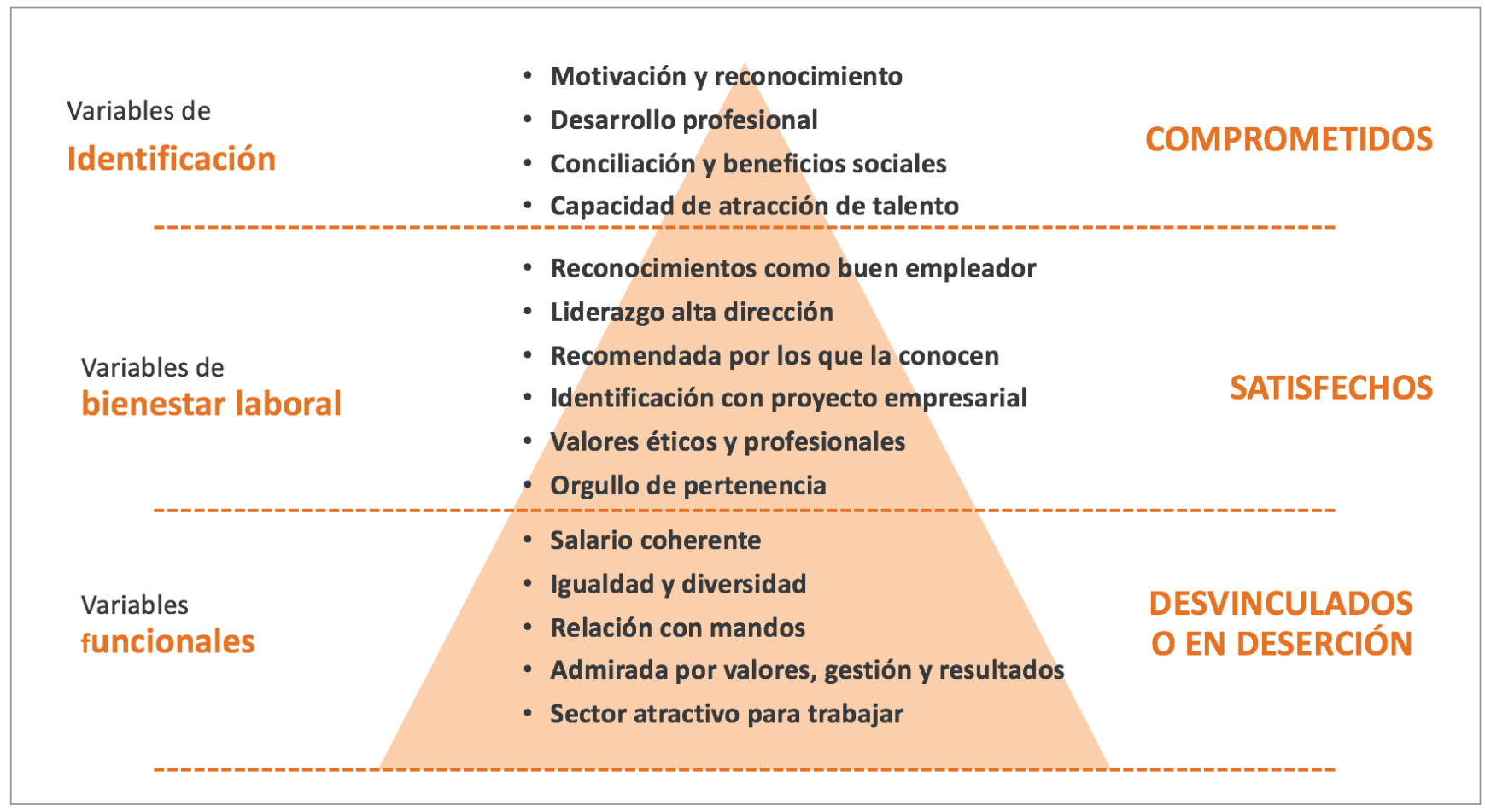

Gráfico 7. La pirámide del compromiso 
sí deberá incluir es una metodología cualitativa que nos descubra el discurso social de los trabajadores respecto a las citadas variables.

\section{Referencias}

Alonso, Álvaro; García-Muina, Fernando-Enrique (2014). "La gestión del talento: líneas de trabajo y procesos clave". Intangible capital, v. 10, n. 5, pp. 1003-1025.

https://doi.org/10.3926/ic.518

Arregle, Jean-Luc; Hitt, Michael; Sirmon, David; Very, Philippe (2007). "The development of organizational social capital: Attributes of family firms". Journal of management studies, v. 44, pp. 73-95.

https://doi.org/10.1111/j.1467-6486.2007.00665.x

Barrow, Simon; Mosley, Richard (2005). The employer brand: Bringing the best of brand management to people at work. Chichester: John Wiley \& Sons. ISBN: 9780470012734 https://goo.gl/Mq1saz

Bartlett, Jennifer; Pallas, Josef; Frostenson, Magnus (2013). "Reputation and legitimacy: accreditation and rankings to assess organizations". In: Carroll, Craig E. The handbook of communication and corporate reputation. Oxford: Wiley Blackwell, pp. 530-544.

https://doi.org/10.1002/9781118335529.ch42

Brown, Michael; Turner, Paul (2008). The admirable company: Why corporate reputation matters so much and what it takes to be ranked among the best. London: Profile Books. ISBN: 9781846680861

Burchell, Michael; Robin, Jennifer (2011). The great workplace: how to build it, how to keep it, and why it matters. San Francisco: Jossey Bass. ISBN: 9780470596265

Caplan, Janice (2011). The value of talent: Promoting talent management across the organization. London: Kogan Page. ISBN: 9780749459840

Carazo-Muriel, José-Antonio (2015). “Análisis y evaluación de los principales modelos de gestión de RRHH". Capital humano, n. 294, pp. 36-39.

http://www.masfamilia.org/files/036_a_CH_Monitores_294. $p d f$

Carreras, Enrique; Alloza, Ángel; Carreras, Ana (2013). Reputación corporativa. Introducción en el carácter científico de la gestión de la reputación corporativa. Madrid: LID Editorial. ISBN: 9788483567210

Castelo-Branco, Manuel; Lima-Rodrigues, Lúcia (2006). "Communication of corporate social responsibility by Portuguese banks: A legitimacy theory perspective". Corporate communications: An international journal, v. 11, n. 3, pp. 232-248.

https://goo.gl/2RCB6J

https://doi.org/10.1108/13563280610680821

Cheese, Peter; Thomas, Robert J.; Craig, Elizabeth (2008). The talent powered organization: strategies for globalization, talent management and high performance. London: Kogan Page. ISBN: 9780749449902

Collings, David G.; Mellahi, Kamel (2009). "Strategic talent management: A review and research agenda". Human resource management review, v. 19, n. 4, pp. 304-313.

https://goo.gl/6FhSG9

https://doi.org/10.1016/j.hrmr.2009.04.001

Cornell, Brandford; Shapiro, Alan (1987) "Corporate stakeholders and corporate finance". Financial management, v. 16, n. 1 , pp. 5-14.

Cubeiro, Juan-Carlos (2003). En un lugar del talento: la sensación de fluidez III. Madrid: Prentice Hall. ISBN: 97884 20537757

Davies, Jacqueline; Kourdi, Jeremy (2010). The truth about talent: A guide to building a dynamic workforce, realizing potential and helping leaders succeed. San Francisco: Jossey Bass. ISBN: 9780470748824

De-la-Fuente-Sabaté, Juan-Manuel; De-Quevedo-Puente, Esther (2003). "Empirical analysis of the relationship between corporate reputation and financial performance: a survey of the literature". Corporate reputation review, v. 6 , n. 2, pp. 161-177.

https://goo.gl/sfUm1v

https://doi.org/10.1057/palgrave.crr.1540197

Fombrun, Charles (2012). "The building blocks of corporate reputation: definitions, antecedents, consequences". En: Pollock, Timothy; Barnett, Michael. The Oxford handbook of corporate reputation, Oxford: Oxford Univ., pp. 94-113. https://doi.org/10.1093/oxfordhb/9780199596706.013.0005

Fombrun, Charles; Shanley, Mark (1990). "What's in a name? Reputation building and corporate strategy". The academy of management journal, v. 33, n. 2, pp. 233-258. https://goo.gl/SB5MVN

Fombrun, Charles; Van-Riel, Cees (1997). "The reputational landscape". Corporate reputation review, v. 1, n. 1, pp. 5-13. https://goo.gl/dzXgww

Fombrun, Charles; Van-Riel, Cees (2003). Fame and fortune: How successful companies build winning reputation. New Jersey: Financial Times Prentice Hall. ISBN: 9780137144419

Freeman, Edward (1984). Strategic management: A stakeholder approach. New York: Pitman. ISBN: 9780273019138

Gasalla, José-María (2015). La nueva dirección de personas. La dirección por confianza $(D p C)$. Madrid: Pirámide. ISBN: 9788436832068

Gratton, Lynda (2003). The democratic enterprise: Liberating your business with freedom, flexibility and commitment. Edinburgh: Financial Times/ Prentice Hall. ISBN: 978 0273675280

Jiménez-Fernández, Alfonso; Aguado-Hernández, Miriam (2009). Employer branding: la gestión de las marcas para atraer y retener talento. Córdoba: Almuzara. ISBN: 97884 92573608

Kehinde, James (2012). "Talent management: Effect on organization performances". Journal of management research, v. 4, n. 2, pp. 178-186.

https://doi.org/10.5296/jmr.v4i2.937

Lange, Donald; Lee, Peggy; Dai, Ye (2011). “Organizational 
reputation: A review". Journal of management, v. 37, n. 1, pp. 153-184.

https://goo.gl/cTp4eb

https://doi.org/10.1177/0149206310390963

Lawler III, Edward (2008). Talent: making people your competitive advantage. San Francisco: Jossey-Bass. ISBN: 9780 787998387

Lewis, Robert; Heckman, Robert (2006). "Talent management: A critical review". Human resource management review, v. 16, n. 2, pp. 139-154

https://goo.gl/fbyNmA

https://doi.org/10.1016/j.hrmr.2006.03.001

Martín-de-Castro, Gregorio; Navas-López, José-Emilio; López-Sáez, Pedro (2006). "Business and social reputation: Exploring the concept and main dimensions of corporate reputation". Journal of business ethics, v. 63, n. 4, p. 362.

https://goo.gl/F8gqJE

https://doi.org/10.1007/s10551-005-3244-z

McWilliams, Abagail; Siegel, Donald (2001). "Corporate social responsibility: A theory of the firm perspective". The academic of management review, v. 26, n. 1, pp. 117-127. https://goo.gl/LKfQNh

McWilliams, Abagail; Siegel, Donald S.; Wright, Patrick M. (2006). "Corporate social responsibility: Strategic implications". Journal of management studies, v. 43, n. 1, pp. 1-18. https://doi.org/10.1111/j.1467-6486.2006.00580.x

Nayar, Vineet (2010). Employees first, customers second: turning conventional management upside down. Boston, Massachusetts: Harvard Business School Press. ISBN: 9781 422139066

Roberts, Peter; Dowling, Grahame (2002). "Corporate reputation and sustained superior financial performance". Strategic management journal, v. 23, n. 12, pp. 1077-1093. https://goo.gl/TKkS19 https://doi.org/10.1002/smj.274

Scullion, Hugh; Collings, David G.; Caligiuri, Paula M. (2010). "Global talent management". Journal of world business, v. 45, pp. 105-108.

https://goo.gl/3qgyGS

https://doi.org/10.1016/j.jwb.2009.09.011
Smart, Bradford D. (1999). Topgrading: How leading companies win by hiring coaching, and keeping the best people. Paramus, NJ: Prentice Hall Press. ISBN: 9780735200494

Stahl, Günter; Björkman, Ingmar; Farndale, Elaine; Morris, Shad; Paauwe, Jaap; Stiles, Philip; Trevor, Jonathan; Wright, Patrick (2012). "Six principles of effective global talent management". MIT Sloan management review, v. 53, n. 2, pp. 25-42. http://sloanreview.mit.edu/article/six-principles-ofeffective-global-talent-management

Tarique, Ibraiz; Schuler, Randall (2010). "Global talent management: Literature review, integrative framework and suggestions for further research". Journal of world business, v. 45, n. 2, pp. 122-133.

https://goo.gl/YfBkMU

https://doi.org/10.1016/j.jwb.2009.09.019

Ulrich, Dave (1996). HR champions: The next agenda for adding value and delivering results. Boston, Massachusetts: Harvard Business School Press.

Ulrich, Dave; Allen, Justin; Brockbank, Wayne; Younger, Jon; Nyman, Mark (2009). HR transformation: Building human resources from the outside in. Chicago: McGraw Hill Professional. ISBN: 9780071664912

Villafañe, Justo (2006). Quiero trabajar aquí. Las seis claves de la reputación interna. Madrid: Pearson Prentice Hall. ISBN: 9788483222935

Villafañe, Justo (2012). La buena empresa. Propuesta para una teoría de la reputación corporativa. Madrid: Gedisa. ISBN: 9788490353066

Walker, Ken (2010). "A systematic review of the corporate reputation literature: Definition, measurement, and theory". Corporate reputation review, v. 12, n. 4, pp. 357-387.

https://goo.gl/quQC7V

https://doi.org/10.1057/crr.2009.26

Walker, James; Larocco, James (2002). "Talent pools: The best and the rest". Human resource planning, v. 25, n. 3, pp. 12-14.

Wartick, Steven (2002). "Measuring corporate reputation". Business and society, v. 41, n. 4, pp. 371-392.

https://doi.org/10.1177/0007650302238774

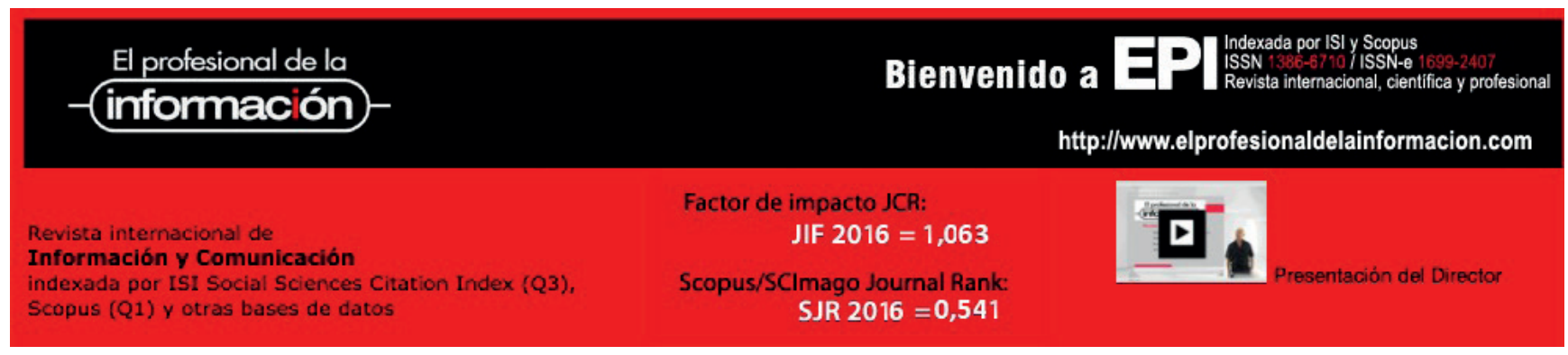




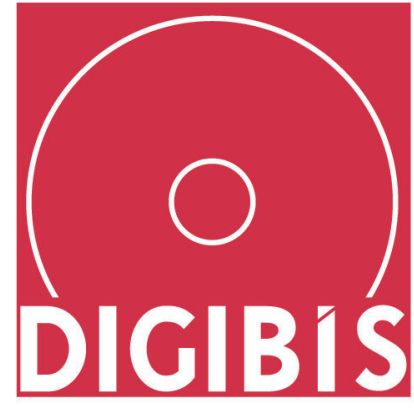

Digitalización enriquecida de fondo antiguo y patrimonial

Software de gestión para Bibliotecas, Archivos y Museos

\author{
DIGIBIB
}

DIGIARCH'

DIGIMÚS

\title{
Recolector OAI-PMH DIGIHUB' de metadatos de diversos proveedores
}

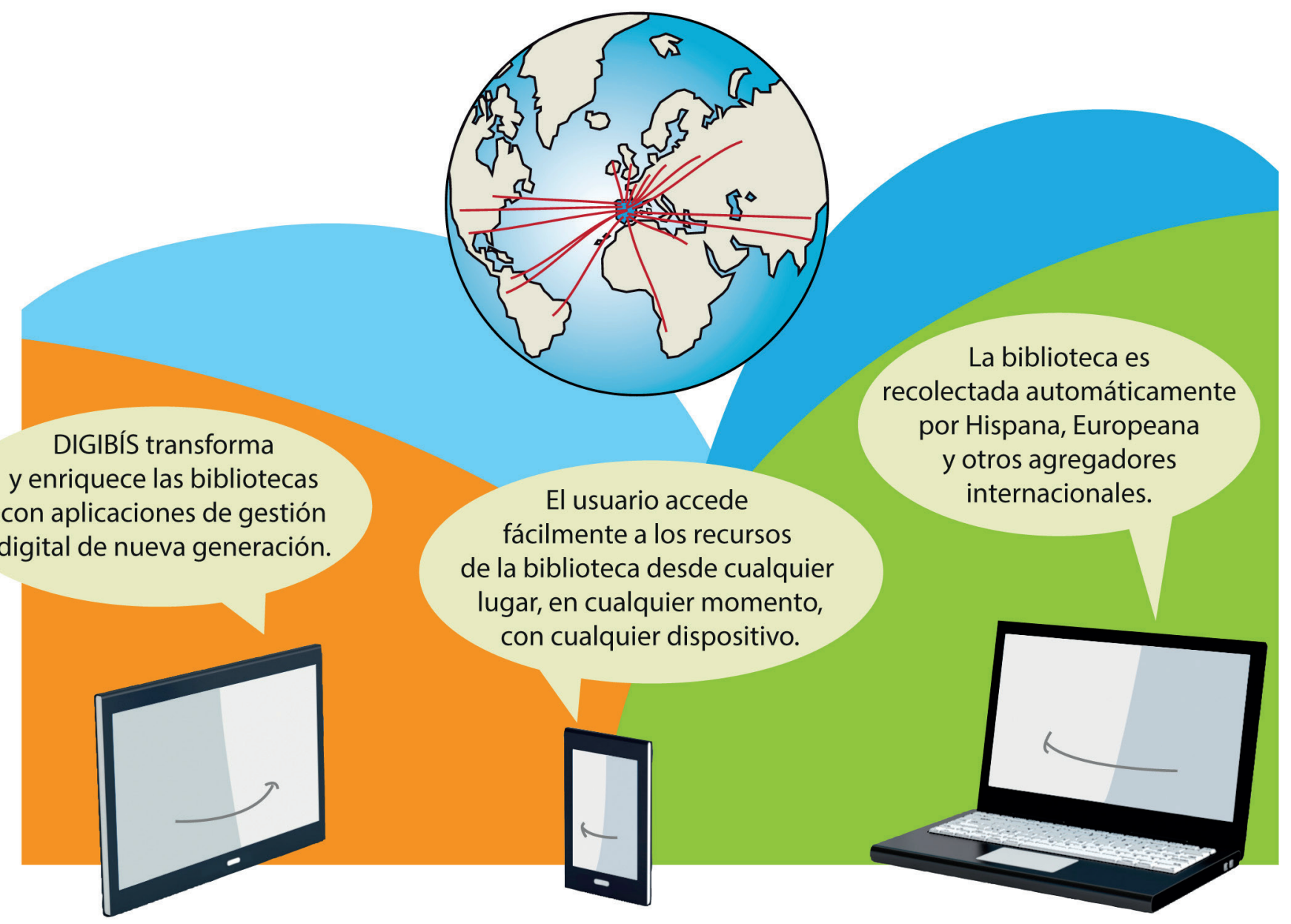

\section{¡Con estándares internacionales para un mundo enlazado y abierto!}

